For, the given identity implies

$$
a_{2 r}+b_{2 r}+\cdots+c_{2 r}=0, \quad(r=0,1, \cdots) ;
$$

and therefore

$$
\begin{gathered}
p_{0}\left(a_{0}+b_{0}+\cdots+c_{0}\right)+p_{2}\left(a_{2}+b_{2}+\cdots+c_{2}\right)+\cdots \\
+p_{2 s}\left(a_{2 s}+b_{2 s}+\cdots+c_{2 s}\right)=0
\end{gathered}
$$

which is the stated conclusion.

California Institute of Technology

\title{
ON SYMMETRIC PRODUCTS OF TOPOLOGICAL SPACES*
}

\section{BY KAROL BORSUK AND STANISLAW ULAM}

1. Introduction. This paper is devoted to an operation that is defined for an arbitrary topological $\dagger$ space $E$ and is analogous to the operation of constructing the combinatorial product spaces. $\ddagger$ We shall be concerned with the topological properties of point sets defined by means of the above operation when executed on the segment $0 \leqq x \leqq 1$.

Let $E$ be an arbitrary topological space. Let $E^{n}$ denote the $n$th topological product of the space $E$, that is, the space whose elements are ordered systems $\left(x_{1}, x_{2}, \cdots, x_{n}\right)$ of points $x_{i} \in E$. By a neighborhood of a point $\left(x_{1}, x_{2}, \cdots, x_{n}\right)$, we understand the set of all systems $\left(x_{1}^{\prime}, x_{2}^{\prime}, \cdots, x_{n}^{\prime}\right)$, where $x_{i}^{\prime}$ belongs to a neighborhood $u_{i}$ of the point $x_{i}$ in the space $E . \ddagger$

The operation with which we are concerned in this paper consists in constructing a space which we shall call the $n$th $s y m$ metric product of the space $E$ and denote by $E(n)$. Its elements are non-ordered systems of $n$ points (which may be different or not) belonging to $E$. Two systems differing only by the order or multiplicity of elements are considered identical. A non-ordered system or simply a set consisting of $n$ points $x_{1}, \cdots, x_{n}$ from the space $E$ will be denoted by $\left\{x_{1}, x_{2}, \cdots, x_{n}\right\}$. If $u_{i}$ is a neighborhood of the point $x_{i}$ in the space $E$, then the set of all systems

* The definition of symmetric products is given below.

$\dagger$ In the sense of Hausdorff, Grundzüge der Mengenlehre, p. 228.

‡ See, for example, F. Hausdorff, Grundzïge der Mengenlehre, p. 102. 
$\left\{x_{1}^{\prime}, x_{2}^{\prime}, \cdots, x_{n}^{\prime}\right\}$ such that $\left\{x_{1}^{\prime}, x_{2}^{\prime}, \cdots, x_{n}^{\prime}\right\} \subset \sum_{i=1}^{n} u_{i}$ and $u_{i} \cdot\left\{x_{1}^{\prime}, x_{2}^{\prime}, \cdots, x_{n}^{\prime}\right\} \neq 0$ for $i=1,2, \cdots, n$ will be, by definition, considered as the neighborhood of the point $\left\{x_{1}, x_{2}, \cdots, x_{n}\right\}$ in the space $E(n)$.

It may be important to observe that the sets $E(n)$ constitute a monotonic sequence of subsets of the space $2^{E}{ }^{*}$ that is, the space whose elements are compact subsets of the space $E . \dagger$ The sets $E(n)$, with finite dimensionality, $\ddagger$ if $E$ is compact and of finite dimensions, approximate in a certain sense the space $2^{E}$; we have, in fact, the following formula:

$$
2^{E}=\overline{\sum_{n=1}^{\infty} E(n) .}
$$

The study of $E(n)$ may, therefore, throw some light on the structure of $2^{E} . \S$

In the case where $E$ is a metric space and $\left|x^{\prime}-x\right|$ denotes the distance of two points $x, x^{\prime} \epsilon E$, we may consider $E^{n}$, and $E(n)$ also, as metric and by means of the following formulas define the distance of two points of these spaces:

$$
\left|\left(x_{1}, x_{2}, \cdots, x_{n}\right)-\left(x_{1}^{\prime}, x_{2}^{\prime}, \cdots, x_{n}^{\prime}\right)\right|=\left(\sum_{i=1}^{n}\left|x_{i}-x_{i}^{\prime}\right|^{2}\right)^{1 / 2}
$$

and

$$
\begin{aligned}
& \left|\left\{x_{1}, x_{2}, \cdots, x_{n}\right\}-\left\{x_{1}^{\prime}, x_{2}^{\prime}, \cdots, x_{n}^{\prime}\right\}\right| \\
& \quad=\operatorname{Max}\left[\operatorname{Sup}_{1 \leqq i \leqq n} \operatorname{Inf}_{1 \leqq j \leqq n}\left|x_{i}-x_{j}^{\prime}\right| ; \operatorname{Sup}_{1 \leqq i \leqq n} \operatorname{Inf}_{1 \leqq j \leqq n}\left|x_{j}-x_{i}\right|\right],
\end{aligned}
$$

respectively. $\Upsilon$

We shall show that, generally speaking, the operations of constructing the combinatorial and the symmetric product of the space $E$ lead to topologically different results. Thus in the

* This very convenient notation was introduced by C. Kuratowski, Fundamenta Mathematicae, vol. 17.

$\dagger$ F. Hausdorff, Grundzüge der Mengenlehre, p. 293.

$\ddagger$ See (b), \$2.

$\S$ The space $2^{E}$ has been recently an object of some studies. See F. Hausdorff, Grundzüge der Mengenlehre, p. 145; S. Mazurkiewicz, Fundamenta Mathematicae, vol. 16, p. 151, and others.

ๆ See Hausdorff, loc. cit. 
case where $E$ is a one-sphere, that is, the circumference of a circle, $E^{2}$ is the surface of an anchor ring (torus) and $E(2)$ is the well known one-sided Möbius strip. Neither of these sets is topologically contained in the other. This example may suffice to show that the symmetric products are apt to be used as means of simple definitions of interesting topological spaces.

In this paper, however, we shall be concerned with the space $I(n), I$ being the segment $0 \leqq x \leqq 1$. The first question to be treated is whether or not $I(n)$ is topologically equivalent with a subset of the $n$-dimensional euclidian space $R^{n}$ ( $R$ is the set of all real numbers).

We formulate our problem in the following manner in order to emphasize certain algebraic analogies.

A real-valued function $\phi\left(x_{1}, x_{2}, \cdots, x_{n}\right)$ of $n$ real variables will be called essentially symmetric if and only if its value depends upon the set $x_{1}, x_{2}, \cdots, x_{n}$ and not upon the order or multiplicity of the $x$ 's. Our problem may now be given the following formulation: Does there exist a system of functions $\phi_{i}\left(x_{1}, x_{2}\right.$, $\left.\cdots, x_{n}\right),(i=1,2, \cdots, n)$, which satisfies the conditions (a) the functions $\phi_{i}$ are essentially symmetric and continuous for $0 \leqq x \leqq 1$; (b) the system of equations $\phi_{i}\left(x_{1}, x_{2}, \cdots, x_{n}\right)=y_{i}$ has at most one solution for every system $y_{1}, y_{2}, \cdots, y_{n}$ ?

We show in $\$ 9$ that the answer to our question is affirmative for $i=1,2,3$ and negative for $i \geqq 4$.

2. Invariants. Let us consider a function $\phi$ defined on the set $E^{n}$ by the formula

$$
\phi\left(x_{1}, x_{2}, \cdots, x_{n}\right)=\left\{x_{1}, x_{2}, \cdots, x_{n}\right\} .
$$

This function transforms $E^{n}$ on $E(n)$ continuously and every point belonging to $E(n)$ is an image of at most $n$ ! points of the set $E^{n}$. If we recall well known properties* of the space $E^{n}$ and continuous transformations, we obtain the propositions:

(a) The properties local-connectedness, separability, compactness, arcwise connectedness, absolute $G_{\delta}$, compactness and local connectedness at once, are invariants under the operation of constructing the symmetric product.

(b) If $E$ is compact, then

* F. G. van Dantzig, Fundamenta Mathematicae, vol. 15, p. 117. 
$\operatorname{Dim} E^{n} \leqq \operatorname{Dim}(E(n)) \leqq \operatorname{Dim} E^{n}+n !-1 \leqq n^{*} \cdot \operatorname{Dim} E+n !-1 . \dagger$

The question whether or not the following topological properties are invariants by the operation of symmetric product remains unsolved:

( $\alpha$ ) To be a locally connected unicoherent continuum.

( $\beta$ ) To possess a fixed point.

( $\gamma$ ) To be an n-dimensional Cantor manifold.

( $\delta)$ To be an absolute retract. $\ddagger$

It may also be interesting to know the exact relation between the dimensions of $E$ and $E(n)$. Finally we wish to find the relation, if any, between the combinatorial characters, that is, the Betti and torsion numbers, of $E$ and $E(n)$.

3. TheOREM 1. Let $A$ be an everywhere dense subset of the dense space $E$. Then $A(n)-E(n-1)=E(n)$ for $n=2,3, \cdots$.

Let $\left\{x_{1}, x_{2}, \cdots, x_{n}\right\} \in E(n)$ and $U_{i}$ be, for $i=1,2, \cdots, n$, any arbitrary neighborhood of the point $x_{i}$ in $E$.

We have to show that there exists a set $\left\{x_{1}^{\prime}, x_{2}^{\prime}, \cdots, x_{n}^{\prime}\right\} \epsilon A$ $(n)-E(n-1)$ such that $\left\{x_{1}^{\prime}, x_{2}^{\prime}, \cdots, x_{n}^{\prime}\right\} \subset \sum_{i=1}^{n} U_{i}$ and

$$
\left\{x_{1}^{\prime}, x_{2}^{\prime}, \cdots, x_{n}^{\prime}\right\} \cdot U_{i} \neq 0 \text { for } i=1,2, \cdots, n \text {. }
$$

The set $A$ constitutes an everywhere dense subset of $E$. Hence for each $i=1, \cdots, n$, there exists a sequence $\left[x_{n}^{(i)}\right]$ consisting of different points of the set $A \cdot U_{i}$. Putting $x_{1}^{\prime}=x_{1}^{(1)}$ and supposing that for a certain $p$ such that $1 \leqq p \leqq n$ the points $x_{2}^{\prime}=x_{k_{p}}^{2)}, \cdots, x_{p}^{\prime}=x_{k_{p}}^{(p)}$ are already defined, we put $x_{p+1}^{1)}$ equal to the first term from the sequence $x_{k}^{(p+1)}$ different from all $x_{i}^{\prime}$. Thus we obtain $n$ different points $x_{1}^{\prime}, x_{2}^{\prime}, \cdots, x_{n}^{\prime} \in A$ or a point $\left\{x_{1}^{\prime}, x_{2}^{\prime}, \cdots, x_{n}^{\prime}\right\}$ from the set $A(n)-E(n-1)$, such that $x_{p}^{\prime}=x_{k_{p}}^{(p)} \epsilon U_{p}$. Hence the theorem is proved.

4. TheOREM 2. The set $I(n)-I(n-1)$, for $n=2,3, \cdots$, is homeomorphic with a subset of $R^{n}$.

Let $T$ be a subset of $R^{n}$ consisting of all points $\left(x_{1}, x_{2}, \cdots, x_{n}\right)$ which fulfill the conditions $0 \leqq x_{1}<x_{2}<\cdots<x_{n} \leqq 1$. For

* W. Hurewicz, Proceedings of the Amsterdam Academy, vol. 30, p. 164.

$\dagger \mathrm{K}$. Menger, Dimensionstheorie, p. 246.

$\ddagger$ A subset $B$ of $A$ is a retract of $A$ if there exists a continuous function $f$, defined on $A$, such that $f(A)=B$ and for every $x \epsilon B, f(x)=x$. An absolute retract, is, by definition, a homeomorph of a retract of the fundamental Hilbert cube. See K. Borsuk, Fundamenta Mathematicae, vol. 17, p. 153 and p. 159. 
$\left(x_{1}, x_{2}, \cdots, x_{n}\right) \epsilon T$ let us put $\psi\left(x_{1}, x_{2}, \cdots, x_{n}\right)=\left\{x_{1}, x_{2}, \cdots, x_{n}\right\}$. It is evident that $\psi$ is a homeomorphism which carries $T$ on $I(n)-I(n-1)$, which was to be proved.

5. Theorem 3. $\operatorname{Dim} I(n)=n$.

In view of 1 (b) and the known fact that $\operatorname{Dim} I^{n}=n$ it will be sufficient to prove that

$$
\operatorname{Dim} I(n) \leqq n .
$$

The truth of inequality (1) is evident if $n=1$, that is, $I(1)$ is identical with the segment $0 \leqq x \leqq 1$. Let us suppose now that for a certain $k$ the inequality (1) is proved. The set $I(k+1)-I(k)$ is open $((\mathrm{a}), \S 2)$; hence it is an $F_{\sigma}$ of dimension at most $k+1$.

Regarding equality $I(k+1)=I(k)+[I(k+1)-I(k)]$ and applying Menger's "Summensatz,"* we obtain the desired inequality.

6. THEOREM 4. Let $A$ denote the segment $I$ without its two ends: $A=I-(0)-(1)$. Then, for each pair of points, $\left\{x_{1}^{\prime}, x_{2}^{\prime}, \cdots, x_{n}^{\prime}\right\}$ and $\left\{x_{1}^{\prime \prime}, x_{2}^{\prime \prime}, \cdots, x_{n}^{\prime \prime}\right\}$ of set $A(n)-I(n-1)$ for $n=2,3, \cdots$, there exists a subset of $A(n)-I(n-1)$ homeomorphic with $I^{n}$ and containing both points.

Proof. We do not diminish the generality if we put

$$
\left\{\begin{array}{l}
0<x_{1}^{\prime}<x_{2}^{\prime}<\cdots<x_{n}^{\prime}<1, \\
0<x_{1}^{\prime \prime}<x_{2}^{\prime \prime}<\cdots<x_{n}^{\prime \prime}<1,
\end{array}\right.
$$

and for a certain $i_{0},\left(1 \leqq i_{0} \leqq n\right)$,

$$
x_{i_{0}}^{\prime}<x_{i_{0}}^{\prime \prime} \text {. }
$$

Let us put for each $\left(t_{1}, t_{2}, \cdots, t_{n}\right) \in R^{n}$

(4) $\left\{\begin{array}{l}x_{i}\left(t_{1}, t_{2}, \cdots, t_{n}\right)=x_{i}^{\prime}+t_{i_{0}}\left(x_{i}^{\prime \prime}-x_{i}^{\prime}\right)+t_{i} \text {, for } i \neq i_{0}, \\ x_{i_{0}}\left(t_{1}, t_{2}, \cdots, t_{n}\right)=x_{i_{0}}+t_{i_{0}}\left(x_{i_{0}}{ }^{\prime \prime}-x_{i_{0}}^{\prime}\right) .\end{array}\right.$

From (2) and (4) it follows that there exists a positive number $\alpha$ such that the inequalities

$$
0 \leqq t_{i_{0}} \leqq 1, \text { and }\left|t_{i}\right| \leqq x
$$

include the inequalities

* See K. Menger, Dimensionstheorie, p. 93. 
(6) $0<x_{1}\left(t_{1}, t_{2}, \cdots, t_{n}\right)<x_{2}\left(t_{1}, t_{2}, \cdots, t_{n}\right)$

$$
<\cdots<x_{n}\left(t_{1}, t_{2}, \cdots, t_{n}\right)<1 .
$$

The points of $R^{n}$ with the coordinates $t_{1}, t_{2}, \cdots, t_{n}$ which satisfy the inequality (5) form in $R^{n}$ a set $P_{n}$ homeomorphic to $I^{n}$. For each point $\left(t_{1}, t_{2}, \cdots, t_{n}\right) \epsilon P_{n}$ let us put

$$
\begin{aligned}
f\left(t_{1}, t_{2}, \cdots, t_{n}\right)=\left\{x_{1}\left(t_{1}, t_{2}, \cdots, t_{n}\right),\right. \\
\left.x_{2}\left(t_{1}, t_{2}, \cdots, t_{n}\right), \cdots, x_{n}\left(t_{1}, t_{2}, \cdots, t_{n}\right)\right\} .
\end{aligned}
$$

This is a continuous function on the compact set $P_{n}$ and, with regard to (3) and (4), a one-to-one correspondence (the second of the equations (4) can be solved for $t_{i_{0}}$, the rest for $t_{i}, i \neq i_{0}$ ). Since the function $f$ carries $P_{n}$ homeomorphically on a subset of the set $A(n)-I(n-1)$ (from (6)) and

$$
\begin{aligned}
f(0,0, \cdots, 0) & =\left\{x_{1}^{\prime}, x_{2}^{\prime}, \cdots, x_{n}^{\prime}\right\}, \\
f(0,0, \cdots, 0,1,0, \cdots, 0) & =\left\{x_{1}^{\prime \prime}, x_{2}^{\prime \prime}, \cdots, x_{n}^{\prime \prime}\right\},
\end{aligned}
$$

our theorem is proved.

7. THEOREM 5. $I(n)$ is an n-dimensional, locally-connected Cantor manifold.*

In view of (a), $\$ 1$, and Theorem 3 , it remains to be shown that no compact $(n-2)$-dimensional subset $C$ from $I(n)$ cuts $I(n)$. Since $I^{n}$ is an $n$-dimensional Cantor-manifold, $\dagger$ it follows from (5) that $A(n)-I(n-1)-C$ is connected and everywhere dense in $A(n)-I(n-1)$. Hence, it follows that from $\$ 3$

$$
\begin{aligned}
A(n)-I(n-1)-C & \subset I(n)-C \subset I(n) \\
& =\overline{A(n)-I(n-1)}=\overline{A(n)-I(n-1)-C,}
\end{aligned}
$$

which includes the fact that $I(n)-C$ is connected.

8. Theorem 6. For $n=1,2,3, I(n)$ is a homeomorph of $I^{n}$.

Proof. Let $\{x, y, z\} \in I(3)$. Since order and multiplicity do not matter, we can suppose that $0 \leqq x \leqq y \leqq z \leqq 1$. Moreover, $x=y$ if and only if $x=y=z$. Let us put

$$
f(\{x, y, z\})=(\xi(x, y, z), \eta(x, y, z), \zeta(x, y, z))
$$

\footnotetext{
* See, for example, K. Menger, Dimensionstheorie, p. 217.
}

$\dagger$ K. Menger, Dimensionstheorie, p. 268. 
where the coordinates $\xi, \eta, \zeta$ of the points $(\xi, \eta, \zeta) \epsilon R^{3}$ are defined by the formulas

$$
\begin{aligned}
& \xi(x, y, z)= \begin{cases}(z-x) \cdot \sin \left(2 \pi \cdot \frac{y-z}{x-z}\right), & \text { if } z>x ; \\
0, & \text { if } z=x ;\end{cases} \\
& \eta(x, y, z)= \begin{cases}(z-x) \cdot \cos \left(2 \pi \cdot \frac{y-z}{x-z}\right), & \text { if } z>x ; \\
0, & \text { if } z>x ;\end{cases} \\
& \zeta(x, y, z)=x .
\end{aligned}
$$

It is easy to observe that $f$ transforms $I(3)$ homeomorphically on a cone $S$, whose base is a circle in the plane $\zeta=0$ with the center $(0,0,0)$ and radius 1 and whose vertex is the point $(0,0,1) ; f(I(2))$ is a triangle with vertices $(0,0,0),(0,1,0)$ and $(0,0,1)$. Finally $f(I(1))$ is a segment $L$ with the ends $(0,0,0)$ and $(0,0,1)$. As a cone is homeomorphic with $I^{3}$ and a triangle with $I^{2}$, we obtain our theorem.

9. TheOREM 7. For $n \geqq 4, I(n)$ is not homeomorphic with any subset of $R^{n}$.

Let $J$ denote the segment $-1 \leqq x \leqq 1$. Noting that $I(n)$ and $J(n)$ are homeomorphic, it will be sufficient to prove that there does not exist a homeomorphism $h$ between $J(n)$ and a subset of $R^{n}$. Let

(11) $Q=E_{\left\{x, y, z, x_{1}, \cdots, x_{n \rightarrow 3}\right\}}[0 \leqq y \leqq z \leqq 1, y=x$ if and only if $x=y=z$,

$$
\text { and } \left.\left|x_{i}+\frac{i}{n}\right| \leqq \frac{1}{3 n} \text { for } 1 \leqq i \leqq n-3\right] \text {. }
$$

Let us say, for each $\left\{x, y, z, x_{1}, x_{2}, \cdots, x_{n-3}\right\} \epsilon Q_{3}$, where $x, y, z, x_{1}, x_{2}, \cdots, x_{n-3}$ are chosen in such a way that the inequalities (11) are satisfied, that

$$
\phi\left(\left\{x, y, z, x_{1}, x_{2}, \cdots, x_{n-3}\right\}\right)=\left(\xi, \eta, \zeta, \xi_{1}, \xi_{2}, \cdots, \xi_{n-3}\right),
$$

where $\xi, \eta, \zeta$ are defined by (8), (9), (10), and

$$
\xi_{i}=x_{i}+\frac{i}{n}, \quad(i=1,2, \cdots, n-3) .
$$

From the definition of $\phi$ and the properties of $f$ expressed by 
(7) it follows immediately that $\phi(Q)$ is a combinatorial product* of $S$ and an $(n-3)$-dimensional product of the segment $[-1 /(3 n)$, $1 /(3 n)]$, that is, topologically a homeomorph of $I^{n}$. The point $f\left(\left\{\frac{1}{2}, \frac{1}{2}, \frac{1}{2}\right\}\right)$ is (by (7), (8), (9) and (10)) an inner point of the cone $S$; it follows that the point

$$
\phi\left(\left\{\frac{1}{2}, \frac{1}{2}, \frac{1}{2},-\frac{1}{n},-\frac{2}{n}, \ldots, \frac{n-3}{n}\right\}\right)
$$

is an inner point of $\phi(Q)$.

Applying Brouwer's theorem $\dagger$ of the invariance of region in $R^{n}$, we conclude that the point $h\left(\frac{1}{2}, \frac{1}{2}, \frac{1}{2},-1 / n,-2 / n, \cdots\right.$, $-(n-3) / n)$ is an inner point of $h(J(n))$. Now let us consider the sequence $\left[p_{k}\right]$ of points from the set $J(n)$ :

$$
p_{k}=\left\{\frac{1}{2}, \frac{1}{2},-\frac{1}{n}+\frac{1}{3 k n},-\frac{1}{n},-\frac{2}{n}, \ldots,-\frac{n-3}{n}\right\} .
$$

We have $p_{k}$ non $\epsilon Q$ and

$$
\lim _{k \rightarrow \infty} p_{k}=\left\{\frac{1}{2}, \frac{1}{2}, \frac{1}{2},-\frac{1}{n},-\frac{2}{n}, \ldots,-\frac{n-3}{n}\right\} .
$$

Noting that $h$ is a homeomorphism, we have further $h\left(p_{k}\right) \in R^{n}$ $-h(Q)$ and

$$
\lim _{k \rightarrow \infty} h\left(p_{k}\right)=h\left(\left\{\frac{1}{2}, \frac{1}{2}, \frac{1}{2},-\frac{1}{n},-\frac{2}{n}, \ldots,-\frac{n-3}{n}\right\}\right),
$$

which is impossible by (13). This proves our theorem.

It may be interesting to know whether or not $I(n)$ is homeomorphic with a subset of $R^{n+1}$.

Lwow, Poland

* See Hausdorff, Mengenlehre.

$\dagger$ L. E. J. Brouwer, Mathematische Annalen, vol. 71, pp. 305-313. 